\title{
Demystifying Genital Prolapse
}

\author{
Philippe Davody* \\ Department of Urology, The American Hospital of Paris, France
}

*Corresponding author: Philippe Davody, Department of Urology, The American Hospital of Paris, France, Tel: (33)145270629;

Email: adavody@icloud.com

Biography: laser-prostate-robot.co.uk or urologie-davody.fr/

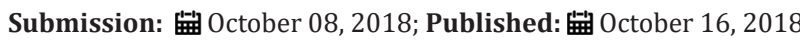

Keywords: Genital prolapse; Pelvic organ prolapse; Organ descent; Uterine prolapse

\section{Editorial}

Genital prolapse, commonly referred to as "organ descent", is a pelvic static disorder that is common in women, usually in the second half of their lives. It is often the result of internal trauma during childbirth, which goes unnoticed and only reveals itself many years later, often during menopause. It very rarely endangers the lives of patients, but it is often responsible for a sometimes major discomfort. Surgical repair, if carried out too late, can leave functional sequelae, particularly neurological, irreversible and which compromise the operated person's perception of the quality of the treatment result, despite a good surgical anatomical result.

\section{What is a genital prolapse?}

Genital prolapse is usually recognized by women as seeing or feeling - especially during intimate hygiene -a bulge of tissue that protrudes to or past the vaginal opening is the most specific symptom. Pelvic organ prolapse, or genital prolapse, is the descent of one or more of the pelvic structures (bladder, uterus, vagina) from the normal anatomic location toward or through the vaginal opening Cystocele is the bulging of the bladder into the vagina, which is most often the case. Enterocele is the bulging of the intestine into the upper part of the vagina. Rectocele is the bulging of the rectum into the vagina. Hysterocele is an abdominal or perineal hernia containing all or part of the uterus.

\section{What is its frequency?}

The exact frequency of pelvis organ prolapse in the female population is difficult to determine and probably underestimated. This prevalence rate can be estimated at around 11\%. In the United Kingdom, genital prolapse surgery accounts for about $20 \%$ of major surgical gynaecological procedures.

Depending on the stage of the perineum affected by the prolapse, the distribution is about $51 \%$ cystocele, $27 \%$ rectocele and $20 \%$ hysterocele. Depending on the age of the patients, the prevalence is around $4 \%$ between 30 and 39 years old, 6\% (between 40 and 49 years old, $12 \%$ between 50 and 59 years old, $12 \%$ between 60 and 69 years old and $11 \%$ between 70 and 79 years old.

\section{Why is this pathology observed in the human species when it is rare if not exceptional in other species?}

Understanding the conditions that cause genital prolapse requires a better understanding of the anatomy of the pelvic floor and perineum in women. Man is the only mammal with a perfectly erect locomotion, which has allowed him to free his upper limbs and use remarkable manual dexterity. This has evolved with brain development and language development.

The development of intelligence has followed the progress of bipedalism. But these mutations in turn led to new anatomical and functional situations that were not always happy. Indeed, erected locomotion has caused fundamental changes in the pelvic skeleton that have evolved in close correlation with those of the muscles and ligaments attached to it.

The resulting new arrangement of the genital, urinary and digestive organs has often been unfavorable compared to that of the animal world, both anatomically and obstetrically. In large quadruped mammals such as dogs, there is no or very rarely genital prolapse, because the pelvic floor is not subjected to the effect of gravity, and the locking by the perineal muscles is, in addition, generally solid Figure 1.

The morphology of the skeleton of the great anthropomorphic "pongidae" apes of Africa or Asia (chimpanzees, gorillas, orangutans), without tails, shows a certain human similarity in the arrangement of the parts that constitute it. However, the posture and bipedal walking of these animals are only occasional and the static mechanics are quite different from those of humans. Prolapse is therefore also rare or unknown here Figure 2.

For women, the erected station and the permanent bipedalism mean that all the forces of gravity are exerted through the small female pelvis. The woman's center of gravity is located at the level indicated by the red dot and moved backwards Figure 3. The effect of body weight on the pelvis has changed the shape of this part of the skeleton over the course of evolution to give it a very characteristic shape specific to the human species. 
One of these characteristics is a very prominent angle between the lumbar spine and the sacrum. This projection is the promontory whose importance as a solid fixation point for surgical repair will be reviewed.

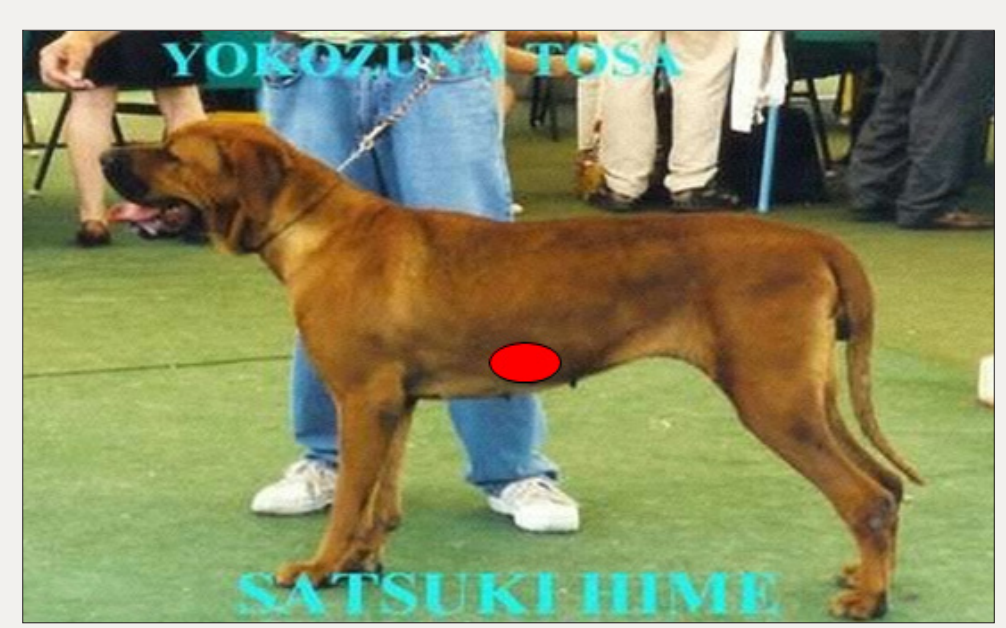

Figure 1:

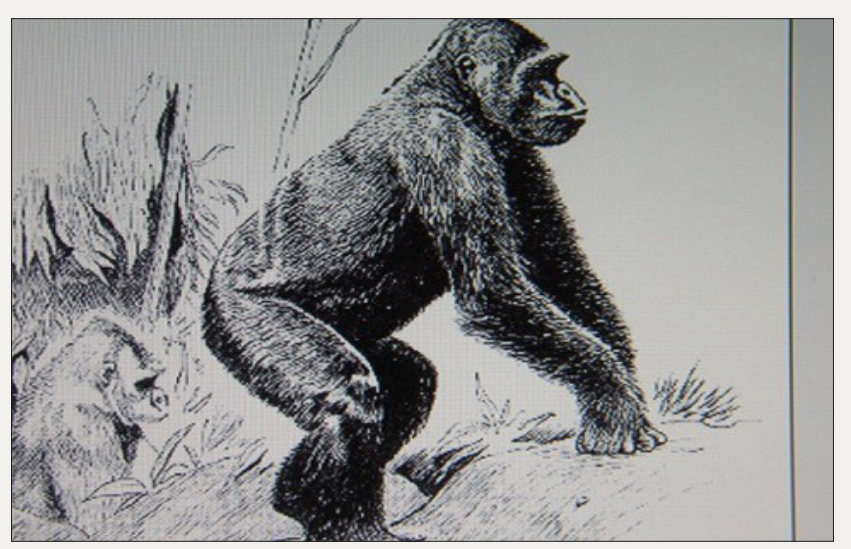

Figure 2:

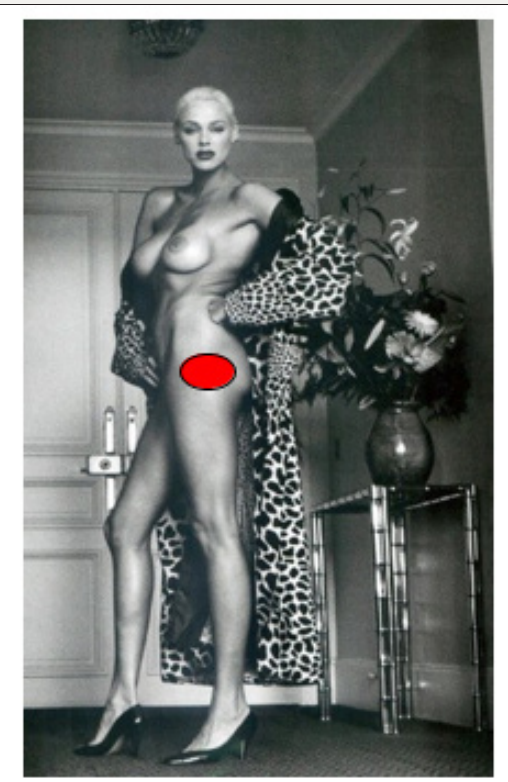

Figure 3: 
The morphology bypassed by this basin, which has become a route crossed by straits, is also very significant.

In humans, obstetrical mechanics can be a problem at the time of delivery, as the dimensions of the pelvic passage are sometimes too small compared to those of the fetus. The presentation, usually the child's head, must engage obliquely in the genital tract, and progression is achieved by precise maneuvers, including "twisting" rotational movements, where the presentation adapts at all times to the diameters most suitable for pelvic excavation.

\section{What are the risk factors for genital prolapse?}

The origin of the development of genital prolapse is certainly multifactorial. The implication of certain risk factors is certain:

1. ageing: the risk doubles every 10 years;

2. the race;

3. the family history;

4. obesity;

5. a great multiparity;

6. a vaginal delivery;

7. constipation.

The role of other factors is advanced, but not formally established:
A. Menopause
B. Some factors related to childbirth:
i. A large fetus;
ii. Longer working hours;
iii. Episiotomy;
iv. Epidural analgesia.

\section{What are the symptoms that can alert you to the presence of genital prolapse?}

Many symptoms are attributed to genital prolapse, but the only specific symptom is the perception of a bulging vaginal area.

The symptoms usually attributed to genital prolapse are:

A. Vaginal symptoms

i. Feeling of a "ball", vaginal bulge or protrusion to the vulva;

ii. Visualization or palpation of a bulge in the vulva or vagina;

iii. Weightlessness or pelvic pain

B. Urinary symptoms

i. Incontinence and urine leaks;

ii. Frequent urination;

iii. Perception of abnormally urgent urination needs;

iv. Weakening of the urinary stream and abnormally prolonged urination;

v. Feeling like emptying the bladder badly after urination

vi. Need to manually reduce prolapse to be able to urinate vii. Need to change position in order to start or complete urination.

C. Digestive symptoms

i. Incontinence for faecal materials or gases;

ii. Feeling of incomplete rectal emptying

iii. Need to produce efforts during defecation, or even to practice digital evacuation;

iv. Need for rectal expression through the posterior vaginal wall.

D. Sexual symptoms

i. Painful or difficult marital relationships

ii. A disappearance of sexual pleasure.

\section{What practical treatment can cure the genital prolapse?}

2.6.1.Simple monitoring: The extent of discomfortand symptoms is not directly proportional to the extent of genital prolapse. This abstention from treatment combined with regular monitoring is justified for moderate prolapse. Some women with severe prolapse prefer not to have surgery, but their monitoring should be closer, especially for symptom worsening or complications such as vaginal erosion, or urinary and/or digestive obstructive disorders.

\section{Non-surgical treatments}

A. Physiotherapy

Pelvic-perineal rehabilitation is effective for the management of female stress urinary incontinence, but not for the treatment of genital prolapse.

B. The pessary

The only effective non-surgical treatment is the wearing of the pessary.

They are widely used, but they are nevertheless restrictive and reserved for women who are "old and have given up...".

\section{Surgical treatments}

The treatment of pelvic static disorders is best addressed through surgery. More than a hundred different surgical procedures have been described to treat them. Classically, the supporters of the vaginal surgery are opposed to the supporters of the abdominal surgery.

The advantage of the vaginal approach is the simplicity of its execution and the comfort of its postoperative suites, but the result usually deteriorates with time and recurrences are frequent. In addition, it can compromise subsequent marital relationships by narrowing the vaginal cavity. The abdominal surgery, through an incision in the lower abdomen, either median from the umbilicus to the pubic bone or transverse, outcomes in a solid and durable repair. It consists of interposing one or two synthetic mesh fixed to the promontory and restoring pelvic statics by a "hammock" effect. This "sacrocolpopexy" is on the other hand more difficult to achieve and its post-operative consequences are more severe. 
The supporters of each approach tried to improve their techniques. The supporters of the vaginal approach worked to improve the strength of their repair, mainly by interposing synthetic mesh under the bladder or in front of the rectum. However, the frequency and severity of rejection of these prostheses by vaginal incisions have resulted in their use being prohibited by the US Food and Drug Administration.

The supporters of the abdominal approached have simplified surgical follow-up, shortened hospitalization and survey times and reduced post-operative pain by replicating the open surgical technique with minimally invasive laparoscopic techniques.
Abdominal incisions are minimal: keyhole incisions. Laparoscopic sacrocolpopexy now appears to be the "gold standard" for the treatment of genital prolapse, especially in young and sexually active women, but progresses in modern anesthesia make it possible to extend its indications widely to older women. Depending on the surgeon's preferences, this laparoscopy can be assisted by a surgical robot which gives the operator a magnification of the vision of the surgical field and a greater precision of the surgical procedure contributing to the quality of the result of the operation Figure 4.

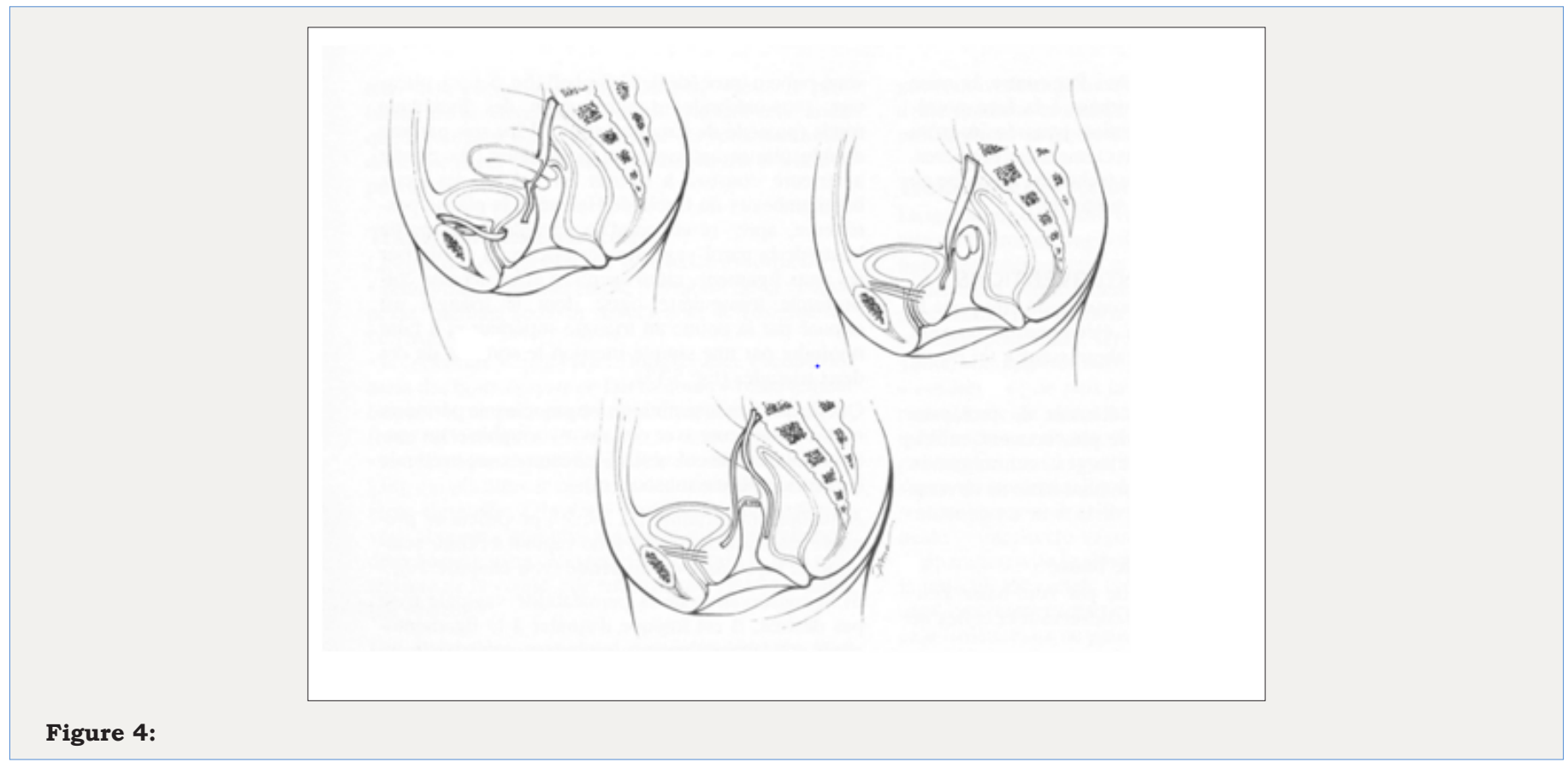

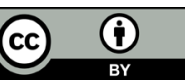

Creative Commons Attribution 4.0 International License

For possible submissions Click Here

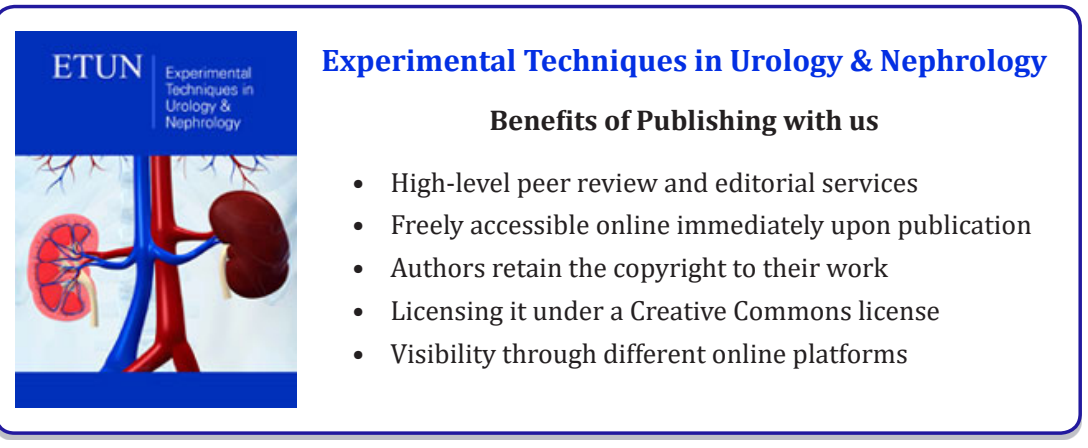

